\title{
An Adaptable Phospholipid Membrane Mimetic System for Solution NMR Studies of Membrane Proteins
}

\author{
Chih-Ta Henry Chien, Lukas R. Helfinger, Mark J. Bostock, Andras Solt, Yi Lei Tan, \\ and Daniel Nietlispach*(1)
}

Department of Biochemistry, University of Cambridge, 80 Tennis Court Road, Cambridge CB2 1GA, U.K.

Supporting Information

ABSTRACT: Based on the saposin-A (SapA) scaffold protein, we demonstrate the suitability of a size-adaptable phospholipid membrane-mimetic system for solution NMR studies of membrane proteins (MPs) under closeto-native conditions. The Salipro nanoparticle size can be tuned over a wide $\mathrm{pH}$ range by adjusting the saposin-tolipid stoichiometry, enabling maintenance of sufficiently high amounts of phospholipid in the Salipro nanoparticle to mimic a realistic membrane environment while controlling the overall size to enable solution NMR for a range of MPs. Three representative MPs, including one Gprotein-coupled receptor, were successfully incorporated into SapA-dimyristoylphosphatidylcholine nanoparticles and studied by solution NMR spectroscopy.

$A$ round $30 \%$ of all genes encode integral membrane $1_{\text {proteins (MPs), }{ }^{1} \text { which have a range of important }}$ biological functions, leading to their involvement in many diseases. Nevertheless, characterization of their structure and dynamics remains heavily under-represented. The biggest challenge for in vitro studies of MPs is finding a suitable mimic of the native lipid membrane bilayer, which is sufficiently stabilizing for structural studies but which maintains the protein's biological function and ability for interaction with ligands and other protein partners. ${ }^{2}$ Detergents are the most commonly used membrane mimetic for MP characterization due to the range available, their simplicity of use, and, for NMR spectroscopy, the relatively small size of the resulting proteindetergent complex. However, detergents may interfere with ligand binding and downstream functional assays, ${ }^{3}$ disrupt protein-protein interactions, ${ }^{4}$ lack crucial protein-lipid contacts, ${ }^{5}$ interfere with protein dynamics, ${ }^{6,7}$ and denature soluble binding partners, ${ }^{8}$ making it difficult to study signaling across the membrane, such as G-protein-coupled receptor (GPCR) signaling. Small bicelles provide a more lipid-like environment. While they may provide high-quality spectra, at sizes suitable for solution NMR there is likely to be rapid mixing between the detergent and lipid components, so this does not provide a pure bilayer environment.

The nanodisc system provides an alternative to detergent micelles and small bicelles for MP studies. The disc-like particles consist of a phospholipid bilayer enclosed by two beltlike membrane-scaffold proteins (MSPs), ${ }^{10}$ with the MP of interest incorporated into the center of the discs. Nanodiscs have been widely used as a detergent-free environment, providing a native-like lipid bilayer suitable for MP characterization. ${ }^{10}$ The suitability of the MSP nanodisc system for solution NMR has been demonstrated, ${ }^{12,13}$ along with the functional relevance of lipid bilayer mimetics for conformational equilibria of receptors and ion channels. ${ }^{14-16}$ However, the relatively large nanodisc particle size is a limitation for solution NMR. Several truncated MSP constructs have been designed to generate smaller nanodiscs, and these modifications facilitated the first solution NMR structure of a MP embedded in phospholipid nanodiscs. ${ }^{7}$ Further improvements have been achieved through the introduction of covalently circularized nanodiscs, which result in a well-defined particle size rather than a broad distribution in sizes that require additional separation through chromatographic methods. ${ }^{17}$ Although MSP nanodiscs have been used successfully in a number of NMR studies, multiple MSP constructs must be tested and the lipid ratio determined empirically for each in order to find the optimal system for every MP of interest, resulting in an extensive screening procedure as demonstrated recently for OprH and OprG. ${ }^{18,19}$ There are concerns that the smaller MSP constructs may be unable to incorporate sufficient lipid molecules with larger MPs to faithfully mimic a native-like membrane bilayer and that some MPs might interact with the scaffold protein. Consequently, alternative nanodisc systems that can adapt to a range of MP sizes are of interest. We describe here applications to solution NMR studies based on the saposin-A (SapA) scaffold protein.

SapA, a sphingolipid activator protein in lysosome, ${ }^{20}$ has been observed to form disc-like particles with liposomes of varying lipid composition at $\mathrm{pH} 4.8 .^{21}$ The crystal structures of SapA revealed a closed conformation in its apo form, which opens in a jack-knife style to interact with a lauryldimethylamine- $N$-oxide (LDAO) mini-bilayer. ${ }^{21}$ Two V-shaped SapAs surrounding the bilayer are in a head-to-tail arrangement, in contrast to a continuous belt found in the MSP nanodiscs. SapA has the potential in this arrangement to incorporate MPs of various sizes by altering the number of SapAs per nanoparticle, removing the need to screen multiple scaffold protein constructs. This idea was confirmed previously in a cryo-electron microscopy (EM) study, where an archaeal mechanosensitive channel T2 $(32.9 \mathrm{kDa})$, which is a putative homopentamer, and a bacterial homotetramer peptide transporter $\mathrm{Pep}_{\mathrm{So} 2}(56 \mathrm{kDa})$ were both incorporated into Salipro nanoparticles at $\mathrm{pH} 7.4 .^{22}$ This study demonstrated that Salipro

Received: June 28, 2017

Published: October 9, 2017 
nanoparticles show promising flexibility, enabling incorporation of a range of protein sizes without the need to screen multiple different scaffold-protein constructs. Thus, Salipro nanoparticles have the flexibility of bicelles, allowing incorporation of a range of protein sizes, but with the benefit of a detergentfree, lipid bilayer environment. This size-adaptability will enable use of the same nanoparticle system for different studies, e.g., cryo-EM studies of a multiprotein complex along with NMR studies of constituent MPs from the complex, facilitating integrated structural studies. Motivated by this, we decided to explore the ability of Salipro nanoparticles to accommodate small to medium size MPs and to test their suitability for solution NMR studies.

In this study, we describe a method to produce SapAdimyristoylphosphatidylcholine (DMPC) nanoparticles with controllable sizes at various $\mathrm{pH}$ values (4.8-7.4) typically used in protein NMR studies. Three model proteins were incorporated into the SapA-DMPC nanoparticles, including a GPCR, showing the broad applicability of this system to MPs of different sizes and folds and at different $\mathrm{pH}$ values. The structural integrity and functionality of the MPs in SapADMPC nanoparticles were characterized by solution NMR spectroscopy.

It has been shown that SapA interacts with liposomes at $\mathrm{pH}$ $4.8,{ }^{21}$ to form disc-like particles, but $n$-dodecyl $\beta$-D-maltoside (DDM)-solubilized lipids are required at neutral $\mathrm{pH} 7.4^{22}$ Here, we investigated the role of detergent in Salipro nanoparticle formation in more detail. Starting with a cloudy DMPC stock solution without DDM in which lipids remain insoluble, SapA was incubated with DMPC in different $\mathrm{pH}$ buffers at $37{ }^{\circ} \mathrm{C}$ for $10 \mathrm{~min}$, and the size of the resulting particles was analyzed by size-exclusion chromatography (SEC). SapA-DMPC nanoparticles with a molecular weight (MW) of $37 \mathrm{kDa}$, which is consistent with the value reported previously, ${ }^{21}$ were formed at $\mathrm{pH} 4.8$ (Figure S1). In contrast, most of the SapA at $\mathrm{pH} 6$ and 7.4 remained the same size as in the apo form, indicating SapA in a closed conformation (Figure S1). We also observed that SapA is insoluble in the absence of DMPC at $\mathrm{pH} 4.8$ (Figure S1). Consequently, we hypothesized that low $\mathrm{pH}$ destabilizes the closed form of SapA, causing either recruitment of DMPC to form Salipro nanoparticles or insolubility in the absence of lipids (Figure S2a). In contrast, SapA remains in the closed conformation at high $\mathrm{pH}$, limiting its ability to form Salipro nanoparticles.

To encourage opening of SapA at higher $\mathrm{pH}$, we investigated the interaction of SapA with DDM, which, unlike lipids, is soluble in water, at $\mathrm{pH}$ 7.4. We observed a significant peak shift in SEC, indicating the formation of a stable SapA-DDM complex (Figure 1a). A blue-shift and the increased intensity in intrinsic fluorescence (Figure 1b), as well as the downfield shift of the tryptophan side-chain signal in $1 \mathrm{D}^{1} \mathrm{H}$ NMR (Figure 1c), observed for SapA-DDM confirm that the environment of the only tryptophan in SapA has become more hydrophobic. Similar but more prominent changes were also observed in SapA-DMPC nanoparticles at $\mathrm{pH} 4.8$ (Figure 1), indicating that SapA adopts an open-like conformation in complex with DDM at $\mathrm{pH}$ 7.4. Therefore, we hypothesized that DDM can promote SapA-DMPC nanoparticle formation by increasing the DMPC solubility and "catalyzing" SapA opening (Figure S2b).

Next, $0.2 \%$ DDM was included in the assembly mixture to assist nanoparticle formation. DDM was removed using detergent adsorbent beads (Figure 2a), and complete detergent removal was confirmed by DDM quantification using the 2,6-
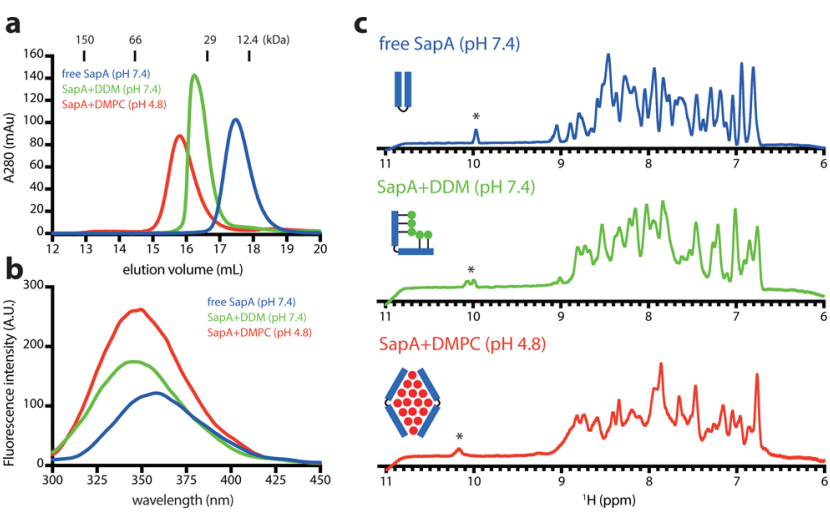

Figure 1. Biophysical characterization of free SapA, SapA-DDM, and SapA-DMPC nanoparticles. (a) Size-exclusion chromatography. (b) Fluorescence spectroscopy. (c) $1 \mathrm{D}{ }^{1} \mathrm{H}$ NMR spectroscopy. Tryptophan indole signals are indicated by $*$.

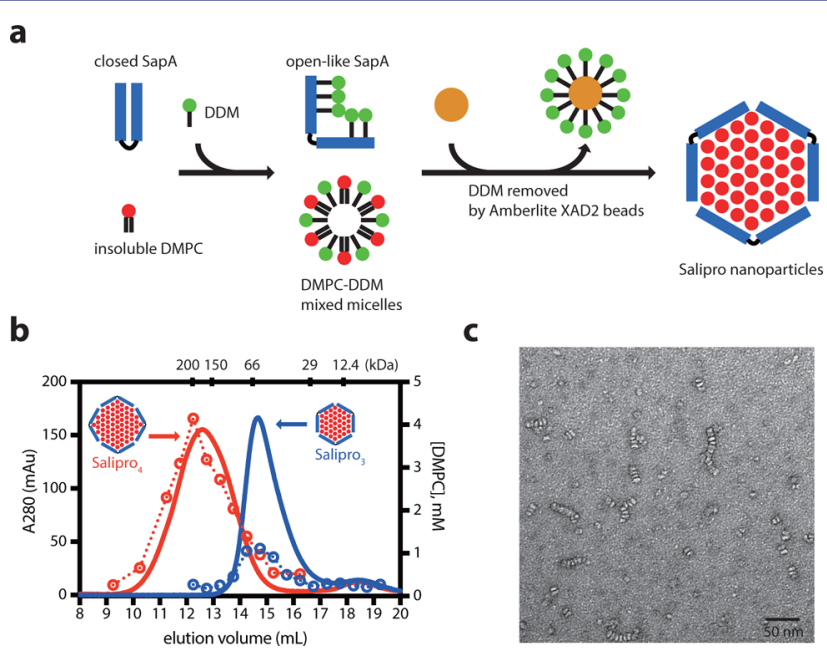

Figure 2. Generation of SapA-DMPC nanoparticles at $\mathrm{pH}$ 6. (a) Schematic representation of the SapA-DMPC nanoparticle assembly. (b) SEC of SapA-DMPC with initial mixing SapA:DMPC ratios of 1:15 (blue) and 1:45 (red) at $\mathrm{pH}$ 6. DMPC was quantified by molybdate assay ${ }^{24}$ (open circle). The proposed SapA arrangement is indicated. (c) Negative-stain EM images of $\mathrm{Salipro}_{3}$. Scale bar, $50 \mathrm{~nm}$.

dimethylphenol method ${ }^{23}$ and 1D ${ }^{1} \mathrm{H}$ NMR (data not shown) to rule out the formation of mixed detergent-lipid Salipro nanoparticles. SEC revealed the formation of two sizes of SapADMPC nanoparticles when different SapA:DMPC ratios were screened at $\mathrm{pH} 6$ (Figure S3a). The optimal assembly SapA:DMPC ratios used to generate a uniform size of Salipro nanoparticles were $1: 15$ and $1: 45$ at $\mathrm{pH}$ 6. The final ratio after SEC was measured using $280 \mathrm{~nm}$ absorbance and molybdate assay, ${ }^{24}$ indicating a ratio of $1: 14$ and 1:45, respectively (Figure $2 \mathrm{~b})$. Based on the MW and the SapA:DMPC ratio, we propose that the small Salipro nanoparticles are composed of three SapA and 42 DMPC molecules, whereas the large Salipro nanoparticles are composed of four SapA and 180 DMPC molecules, hereafter called Salipro ${ }_{3}$ and $\mathrm{Salipro}_{4}$, respectively. The disc-like shape is confirmed by negative-stain EM (Figures $2 \mathrm{c}$ and S3d); a side view of the Salipro nanoparticles stacked together can be seen, as well as a top view of single Salipro nanoparticles. Stacking is also observed in EM images of MSP nanodiscs. ${ }^{25}$ The same size-tunable characteristic was also found at $\mathrm{pH} 4.8$ and 7.4 (Figure S3b,c). SapA can also form Salipro nanoparticles when mixtures of lipids are used, as 
shown, e.g., for DMPC/DMPG, resulting in homogeneous particles similar in size to the ones with pure DMPC (Figure S4).

To test whether SapA-DMPC nanoparticles can be used in MP NMR studies, we first investigated the incorporation of the $16.5 \mathrm{kDa}$ bacterial outer membrane protein X (OmpX). OmpX embedded in phospholipid MSP nanodiscs has previously been studied extensively by NMR spectroscopy, providing a good point of comparison. $6,7,26$

The self-assembly of OmpX embedded in SapA-DMPC nanoparticles was achieved by mixing SapA, DMPC, and DPCsolubilized OmpX at a ratio of 10:50:1, at $\mathrm{pH}$, with $0.2 \%$ DDM to facilitate opening of SapA, followed by a detergent removal step. OmpX remained in solution and showed a symmetrical peak at $14.1 \mathrm{~mL}(84 \mathrm{kDa})$ by SEC (Figure S5), slightly smaller than that of OmpX in MSP1D1 $\Delta \mathrm{H} 5(\Delta \mathrm{H} 5)$ nanodiscs $(107 \mathrm{kDa}){ }^{7}$ We suggest that $\mathrm{OmpX}$ was incorporated into Salipro $_{3}$. A 2D $\left[{ }^{1} \mathrm{H}^{15} \mathrm{~N}\right]$-SOFAST-TROSY spectrum was recorded on non-deuterated ${ }^{15} \mathrm{~N}$-labeled OmpX (Figure 3) to assess the structural integrity of OmpX in

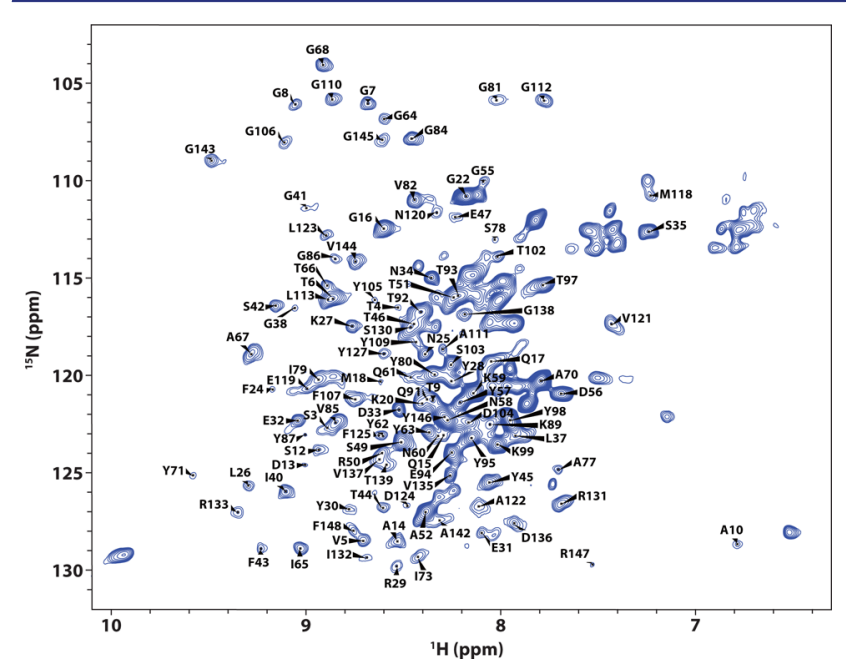

Figure 3. 2D $\left[{ }^{1} \mathrm{H},{ }^{15} \mathrm{~N}\right]$-SOFAST-TROSY spectrum of non-deuterated ${ }^{15} \mathrm{~N}$-labeled OmpX in SapA-DMPC nanoparticles.

Salipro $_{3}$. The resulting spectrum is of good quality, even without deuteration, and, with regard to the peak positions, very similar in appearance to a previously presented spectrum of OmpX in $\Delta \mathrm{H} 5$-DMPC nanodiscs, allowing transfer of the reported backbone assignments on the basis of peak proximity (assignments kindly provided by Dr. Stefan Bibow). ${ }^{6}$ Clearly, the spectrum quality for the latter in $\triangle \mathrm{H} 5-\mathrm{DMPC}$ nanodiscs is superior due to the use of deuterated OmpX. ${ }^{26}$ However, rotational correlation time measurements using ${ }^{15} \mathrm{~N}$ TRACT ${ }^{27}$ indicate that the Salipro nanoparticle-embedded OmpX is smaller in size $\left(\tau_{\mathrm{c}}=28 \mathrm{~ns}\right.$, calculated $\left.\mathrm{MW}=87 \mathrm{kDa}\right)$ compared to the $\Delta \mathrm{H} 5$ nanodiscs $\left(\tau_{\mathrm{c}}=34 \mathrm{~ns}\right.$, calculated MW $\left.=107 \mathrm{kDa}\right)$. Accordingly, using deuterated protein would result in highquality spectra in Salipro nanoparticles. A chemical-shift-based comparison of $\mathrm{OmpX}$ in $\mathrm{Salipro}_{3}$, with the same protein embedded in $\triangle \mathrm{H} 5-\mathrm{DMPC}^{6}$ or in DPC micelles, ${ }^{7}$ confirms the DMPC bilayer environments of OmpX to be most similar to $\mathrm{Salipro}_{3}$ (Figure S6). This supports the proper incorporation of the protein into $\mathrm{Salipro}_{3}$. Interestingly, the residues with the largest chemical shift differences between the SapA-DMPC nanoparticles and SapA-DMPC/DMPG nanoparticles mostly reside in loops 1 and 3 (Figure $S 6 c, d$ ), indicating that the phospholipid headgroup composition has a strong influence on specific regions of the MP.

To explore the more general suitability of the Salipro nanoparticle system, the incorporation of other MPs with different structural folds was investigated. A seven-transmembrane helical protein, the phototaxis receptor sensory rhodopsin II (pSRII) (26.4 kDa), was successfully incorporated using the same protocol and ratio. UV-vis analysis confirmed the native conformation of pSRII with its characteristic $498 \mathrm{~nm}$ absorbance. SEC and negative-stain EM images show homogeneous particles with $\mathrm{MW} \approx 200 \mathrm{kDa}$ (Figure S7); thus, we suggest that pSRII was embedded in Salipro 4 . Based on UV-vis and SDS-PAGE quantitative analyses, the SapA:pSRII ratio was determined to be 2:1 (Figure S8), suggesting dimeric pSRII in Salipro $_{4}$. A $2 \mathrm{D}\left[{ }^{1} \mathrm{H},{ }^{15} \mathrm{~N}\right]$-SOFASTTROSY spectrum (Figure S9) showed that although the NMR spectral quality is worse compared to that of monomeric pSRII in c7-DHPC micelles, ${ }^{28}$ as expected due to the larger MW, a similar overall backbone fingerprint can be observed. Perdeuteration would further improve the spectral quality for more extensive solution NMR studies of pSRII in SapA-DMPC nanoparticles.

Next, we used thermostabilized turkey $\beta_{1}$-adrenergic receptor $\left(\beta_{1} \mathrm{AR}\right)(36 \mathrm{kDa})$, a GPCR, to test if MPs in the SapA-DMPC nanoparticles retains the ability to functionally interact with binding partners. It has been shown previously that $\beta_{1} \mathrm{AR}$ interacts with the full agonist isoprenaline and the G-protein mimicking nanobody $\mathrm{Nb80}$ using detergent micelles. ${ }^{29}$ Here, we incorporated $\left[{ }^{13} \mathrm{C}_{\varepsilon}\right.$-methionine $] \beta_{1} \mathrm{AR}$ (Figure S10) into SapA-DMPC nanoparticles at $\mathrm{pH} 7.4$ (Figure S11) due to the limited stability of $\beta_{1} \mathrm{AR}$ at low $\mathrm{pH}$. The ${ }^{13} \mathrm{C}$ HMQC spectrum of $\beta_{1} \mathrm{AR}$ in the apo state revealed 13 peaks for the 12 methionine residues, with substantially varying intensities, indicating that diverse parts of the receptor apo form differ in their dynamics. Several signals showed chemical shift perturbation upon isoprenaline binding (Figure 4, arrows), indicating that the receptor changes conformation when bound to an activating ligand. The substantial broadening observed for many of the NMR signals in the full agonist bound state further suggests that the receptor is dynamically exchanging between several conformations on a $\mu$ s-to-ms time scale. Addition of G-

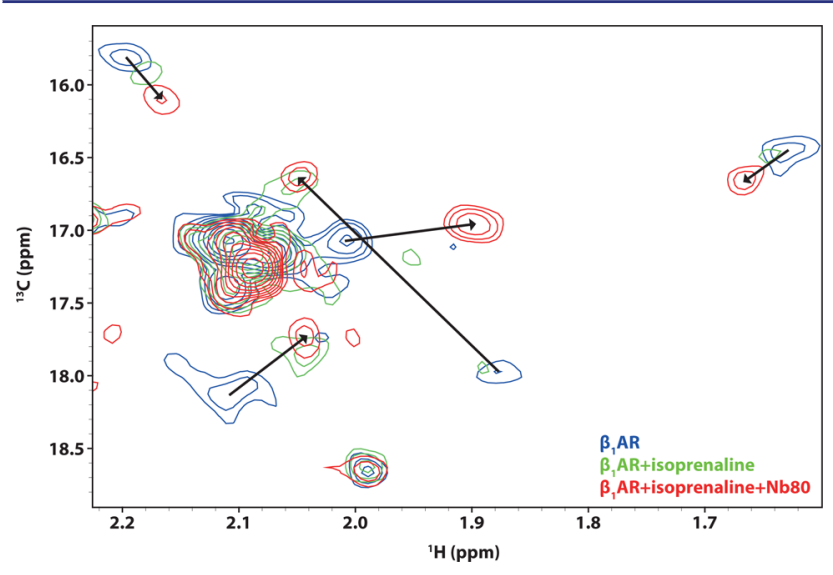

Figure 4. Overlay of the $2 \mathrm{D}{ }^{13} \mathrm{C}$-SOFAST-HMQC spectra of $\left[{ }^{13} \mathrm{C}_{\varepsilon^{-}}\right.$ methionine] $\beta_{1} \mathrm{AR}$ in the apo state (blue), the isoprenaline-bound state (green), and the isoprenaline-Nb80-bound ternary complex (red). 
protein mimicking $\mathrm{Nb} 80$ to form a fully active ternary complex induced further dramatic chemical shift perturbations. At the same time the recovery of peak intensities seems to indicate that the isoprenaline-Nb80-bound ternary complex is conformationally more restricted, comparable to NMR observations for the $\beta_{2}$-adrenergic receptor. ${ }^{30}$ While these studies show the successful embedding of $\beta_{1} \mathrm{AR}$ in Salipro nanoparticles as a functional receptor, the individual resonance assignments will be presented as the topic of further investigations.

In conclusion, we have presented a flexible phospholipid nanodisc system based on the SapA scaffold protein which can accommodate MPs with different fold and size over a wide $\mathrm{pH}$ range and allows interaction with protein binding partners to be studied. Based on the three proteins studied here, we observe high incorporation efficiencies into Salipro nanoparticles of $>80 \%$. Furthermore, Salipro nanoparticle NMR samples were stable over extended periods of time (Figure S13). The compatibility of the SapA system with NMR spectroscopy provides an opportunity to study MPs' structure, dynamics, and molecular interactions, such as GPCR signaling in a more native lipid environment, demonstrating the potential of the SapA nanodisc system for the MP field.

\section{ASSOCIATED CONTENT}

\section{S Supporting Information}

The Supporting Information is available free of charge on the ACS Publications website at DOI: 10.1021/jacs.7b06730.

Experimental details and additional data, including Figures S1-S13 (PDF)

\section{AUTHOR INFORMATION}

\section{Corresponding Author}

*dn206@cam.ac.uk

\section{ORCID}

Daniel Nietlispach: 0000-0003-4364-9291

\section{Funding}

This work was funded in part through a BBSRC research grant to D.N. (BB/K01983 X/1), studentship support to C.H.C. (Taiwan Cambridge Scholarship), and studentship support to A.S. (MRC Industrial CASE).

\section{Notes}

The authors declare no competing financial interest.

\section{ACKNOWLEDGMENTS}

We thank Dr. Janet Deane for providing the SapA plasmid and Dr. Dijun Du for help with EM. NMR resonance assignments of OmpX in MSP1D1 $\mathrm{H}$ 5-DMPC nanodiscs were kindly provided by Dr. Stefan Bibow. We thank the BBRSC, Taiwan Cambridge Scholarship and MRC for funding.

\section{REFERENCES}

(1) Wallin, E.; von Heijne, G. Protein Sci. 1998, 7, 1029.

(2) Cross, T. A.; Murray, D. T.; Watts, A. Eur. Biophys. J. 2013, 42, 731.

(3) Ding, Y.; Fujimoto, L. M.; Yao, Y.; Plano, G. V.; Marassi, F. M. Biochim. Biophys. Acta, Biomembr. 2015, 1848, 712.

(4) Zhang, M.; Huang, R.; Im, S. C.; Waskell, L.; Ramamoorthy, A. J. Biol. Chem. 2015, 290, 12705.

(5) Laganowsky, A.; Reading, E.; Allison, T. M.; Ulmschneider, M. B.; Degiacomi, M. T.; Baldwin, A. J.; Robinson, C. V. Nature 2014, 510, 172.
(6) Frey, L.; Lakomek, N. A.; Riek, R.; Bibow, S. Angew. Chem., Int. Ed. 2017, 56, 380.

(7) Hagn, F.; Etzkorn, M.; Raschle, T.; Wagner, G. J. Am. Chem. Soc. 2013, 135, 1919.

(8) Warschawski, D. E.; Arnold, A. A.; Beaugrand, M.; Gravel, A.; Chartrand, É.; Marcotte, I. Biochim. Biophys. Acta, Biomembr. 2011, 1808, 1957.

(9) Poget, S. F.; Girvin, M. E. Biochim. Biophys. Acta, Biomembr. 2007, 1768,3098

(10) Bayburt, T. H.; Grinkova, Y. V.; Sligar, S. G. Nano Lett. 2002, 2, 853.

(11) Denisov, I. G.; Sligar, S. G. Chem. Rev. 2017, 117, 4669.

(12) Raschle, T.; Hiller, S.; Yu, T.-Y.; Rice, A. J.; Walz, T.; Wagner, G. J. Am. Chem. Soc. 2009, 131, 17777.

(13) Etzkorn, M.; Raschle, T.; Hagn, F.; Gelev, V.; Rice, A. J.; Walz, T.; Wagner, G. Structure 2013, 21, 394.

(14) Imai, S.; Osawa, M.; Mita, K.; Toyonaga, S.; Machiyama, A.; Ueda, T.; Takeuchi, K.; Oiki, S.; Shimada, I. J. Biol. Chem. 2012, 287, 39634.

(15) Minato, Y.; Suzuki, S.; Hara, T.; Kofuku, Y.; Kasuya, G.; Fujiwara, Y.; Igarashi, S.; Suzuki, E.-I.; Nureki, O.; Hattori, M.; Ueda, T.; Shimada, I. Proc. Natl. Acad. Sci. U. S. A. 2016, 113, 4741.

(16) Kofuku, Y.; Ueda, T.; Okude, J.; Shiraishi, Y.; Kondo, K.; Mizumura, T.; Suzuki, S.; Shimada, I. Angew. Chem., Int. Ed. 2014, 53, 13376.

(17) Nasr, M. L.; Baptista, D.; Strauss, M.; Sun, Z.-Y. J.; Grigoriu, S.; Huser, S.; Plückthun, A.; Hagn, F.; Walz, T.; Hogle, J. M.; Wagner, G. Nat. Methods 2017, 14, 49.

(18) Viegas, A.; Viennet, T.; Etzkorn, M. Biol. Chem. 2016, 397, 1335.

(19) Kucharska, I.; Edrington, T. C.; Liang, B.; Tamm, L. K. J. Biomol. NMR 2015, 61, 261.

(20) Kolter, T.; Sandhoff, K. FEBS Lett. 2010, 584, 1700.

(21) Popovic, K.; Holyoake, J.; Pomès, R.; Privé, G. G. Proc. Natl. Acad. Sci. U. S. A. 2012, 109, 2908.

(22) Frauenfeld, J.; Löving, R.; Armache, J.-P.; Sonnen, A. F.-P.; Guettou, F.; Moberg, P.; Zhu, L.; Jegerschöld, C.; Flayhan, A.; Briggs, J. A. G.; Garoff, H.; Löw, C.; Cheng, Y.; Nordlund, P. Nat. Methods 2016, 13, 345.

(23) Prince, C.; Jia, Z. Acta Crystallogr., Sect. D: Biol. Crystallogr. 2012, 68, 1694

(24) Prince, C.; Jia, Z. Methods Enzymol. 2015, 557, 95.

(25) Hopper, J. T. S.; Yu, Y. T.-C.; Li, D.; Raymond, A.; Bostock, M.; Liko, I.; Mikhailov, V.; Laganowsky, A.; Benesch, J. L. P.; Caffrey, M.; Nietlispach, D.; Robinson, C. V. Nat. Methods 2013, 10, 1206.

(26) Hagn, F.; Wagner, G. J. Biomol. NMR 2015, 61, 249.

(27) Lee, D.; Hilty, C.; Wider, G.; Wüthrich, K. J. Magn. Reson. 2006, 178,72 .

(28) Gautier, A.; Mott, H. R.; Bostock, M. J.; Kirkpatrick, J. P.; Nietlispach, D. Nat. Struct. Mol. Biol. 2010, 17, 768.

(29) Isogai, S.; Deupi, X.; Opitz, C.; Heydenreich, F. M.; Tsai, C.-J.; Brueckner, F.; Schertler, G. F. X.; Veprintsev, D. B.; Grzesiek, S. Nature 2016, 530, 237.

(30) Nygaard, R.; Zou, Y.; Dror, R. O.; Mildorf, T. J.; Arlow, D. H.; Manglik, A.; Pan, A. C.; Liu, C. W.; Fung, J. J.; Bokoch, M. P.; Thian, F. S.; Kobilka, T. S.; Shaw, D. E.; Mueller, L.; Prosser, R. S.; Kobilka, B. K. Cell 2013, 152, 532. 